\title{
Sound scattering by spherical and elongated shelled bodies
}

\author{
T.K. Stanton \\ Department of Applied Ocean Physics and Engineering, Woods Hole Oceanographic Institution, \\ Woods Hole, Massachusetts 02543
}

(Received 11 October 1989; accepted for publication 15 May 1990)

\begin{abstract}
Describing the scattering of sound by elongated objects with high aspect ratios (ratio of length to diameter) usually involves great numerical difficulties. The recently developed deformed cylinder solution was shown to be increasingly accurate in the limit of very high aspect ratios ( $\gtrsim 5: 1$ ) while requiring relatively low computation times and was applied to objects of constant composition [T. K. Stanton, "Sound scattering by cylinders of finite length. III. Deformed cylinders," J. Acoust. Soc. Am. 86, 691-705 (1989) ]. In this article, the approximate formulation is used to describe scattering by prolate spheroids, straight finite cylinders, and uniformly bent cylinders where the objects are composed of an elastic shell surrounded by fluid and filled with either a fluid or gas. The calculations are compared with those involving spherical shells based on the formulation derived in Goodman and Stern [J. Acoust. Soc. Am. 34, 338-344 (1962)]. The calculations are made over a wide range of frequencies and shell thicknesses (ranging from solid elastic objects to thin-shelled objects). Since the deformed cylinder formulation is most accurate for angles of incidence normal or near normal to the lengthwise axis, the calculations are limited to broadside incidence. The simulations show significant variations in the modal interference structure as the shell thickness and shape are varied. Comparisons are also made between predictions and laboratory data involving straight and bent finite-length cylindrical shells (stainless steel) with 3:1 aspect ratios and 52\% shell thicknesses. The study not only shows reasonable agreement between the predictions and data, but also illustrates the dramatic change in scattering cross section due to the bend of the object ( $12 \mathrm{~dB}$ in this case).
\end{abstract}

PACS numbers: 43.20.Fn, 43.30.Gv

\section{INTRODUCTION}

The scattering of sound by elastic shelled bodies has been studied by many investigators over the past several decades. The vast majority of the work has involved spherical shells ${ }^{1-6}$ and infinitely long cylindrical shells, ${ }^{6-17}$ while there are very few publications describing finite length shelled objects $^{17-20}$ (the reason being the lack of an exact theory and the computational difficulties involved in the numerical approaches for finite objects). One numerical approach, the $T$ matrix method, has been used to describe the scattering by finite elongated objects ${ }^{17-20}$ and Hackman and Todoroff have recently reduced its convergence problems involving high aspect objects by use of a spheroidal-based coordinate system. ${ }^{20}$ Hackman is currently applying their formulation to the scattering by high-aspect prolate spheroid shells. ${ }^{21}$

In a recent series of articles, we developed an approximate analytical solution that can be used to describe the scattering of sound by elongated objects of high aspect. ${ }^{22-24}$ The generalized approach described in the third article of the series, the "deformed cylinder" formulation, allows one to calculate the scattered field of elongated objects whose properties such as the cross-sectional radius and material composition may vary with respect to the lengthwise axis. In addition, the axis of the cylinder may be deformed. The approximation lies in the assumption that the boundary con- ditions at each position along the axis are the same as those of the infinitely long cylinder; i.e., each infinitesimal section of the cylinder behaves as if the neighboring sections are identical where, in fact, their properties are slowly varying. As a result, the formulation is only valid for angles of incidence and reception normal or nearly normal to all tangents of the axis and becomes increasingly accurate for objects in the limit of high aspect ratios ( $\gtrsim 5: 1)$. In that article, there was excellent agreement between the deformed cylinder calculations and the exact spheroidal wave function solution when the scattering by prolate spheroids (aspect ratio of $5: 1$ ) was calculated.

While the deformed cylinder formulation is approximate and has certain limitations, it has distinct advantages over other approaches. While the numerical approaches tend to have convergence difficulties as the aspect ratio of the objects is increased, the deformed cylinder formulation becomes increasingly accurate as the ratio is increased. Furthermore, since its boundary conditions are simple, involving those of the infinitely long cylinder, the computation time is relatively small. For example, in the previously mentioned example involving prolate spheroids, the deformed cylinder.calculations were 100 times faster than the calculations using the exact spheroidal wave function solution. Thus when computation time using other techniques becomes prohibitively long for high aspect objects, it is advan- 
tageous to consider the deformed cylinder formulation.

In our recent studies, we have sought solutions to describe the scattering by marine organisms. ${ }^{22,24,25}$ The deformed cylinder solution was developed to account for the body shape and variable material properties of the organisms. The uniformly bent finite cylinder appeared to be a reasonable "first-order" approximation for the description of euphausiids (a shrimplike marine organism). However, the material of the organisms was assumed to be $100 \%$ fluid. Since many organisms including euphausiids are covered with an elastic shell, the fluid body assumption may not be valid. The scattering data from the organisms that we analyzed were not of the quality required to differentiate between an elastic shelled body and a fluid-only body. Thus it is quite possible that the "effective" fluid material properties of the organisms discussed in those articles actually had an appreciable elastic shell component to them. For these reasons and the fact that there is a general need for such computations, we now study the scattering by elongated shelled objects.

In this article, we use the recently developed deformed cylinder solution to describe sound scattering by high aspect ratio ( $\gtrsim 5: 1$ ) shelled bodies: prolate spheroid, straight finite cylinder, and uniformly bent finite cylinder. The calculations are compared with results involving scattering by spherical shells as derived in a manner similar to that described by Goodman and Stern. ${ }^{1}$ In each case, the object is composed of an elastic shell (stainless steel) filled with either a fluid or gas (air) and is surrounded by a fluid (water). The calculations are made over a wide range of frequencies and shell thicknesses (ranging from solid elastic objects to thin-shelled objects). Since the deformed cylinder formulation is most accurate for angles of incidence normal or near normal to the lengthwise axis, the calculations were restricted to only normal incidence. Comparisons are also made between predictions and laboratory data involving straight and bent finite-length cylindrical shells with $3: 1$ aspect ratios and $52 \%$ shell thicknesses.

\section{THEORY}

In this section, we summarize the general solutions that we will use to calculate the scattering by spheres, straight finite cylinders, prolate spheroids, and uniformly bent cylinders. The derivations for the solutions appear in other articles (all objects but the sphere involve use of the deformed cylinder solution ). Since the modal series coefficients for the shelled body case for the sphere (spherical boundary conditions) and all (high-aspect ratio) elongated objects (cylindrical boundary condition approximation) already exist in the literature, those coefficients will simply be referenced to, rather than rederived.

The scattered pressure field can be expressed in terms of the scattering amplitude $f$ as

$$
p_{\text {scat }}=P_{0}\left(e^{i k r} / r\right) f(\Omega),
$$

where $P_{0}$ is the amplitude of the incident plane wave $i=\sqrt{-1}, k$ is the acoustic wave number of the surrounding fluid $(=2 \pi / \lambda)$, and $r$ is the range to the object from the field point (receiver). The differential scattering cross section is then

$$
\sigma(\Omega) \equiv|f(\Omega)|^{2}
$$

and the (differential) backscattering cross section (monostatic) as defined by Clay and Medwin ${ }^{26}$ is

$$
\sigma_{b s}=\left|f\left(\Omega_{b s}\right)\right|^{2} \text {. }
$$

The target strength is defined in Urick as ${ }^{27}$

$$
\mathrm{TS} \equiv 10 \log \left(R^{2} I_{b s} / I_{0}\right)=10 \log \left|f\left(\Omega_{b s}\right)\right|^{2}=10 \log \sigma_{b s},
$$

where $I_{0}$ is the intensity of the incident plane wave and $I_{b s}$ is the intensity of the backscattered field measured at a reference distance $R$.

\section{A. Spherical shell-exact solution}

The general solution to the scattering by a spherical object is given $a^{28}$

$$
\begin{aligned}
p_{\text {scat }} & =P_{0} \sum_{m=0}^{\infty} i^{m}(2 m+1) b_{m} P_{m}(\cos \theta) h_{m}^{(1)}(k r) \\
& \rightarrow P_{0} \frac{e^{i k r}}{r}\left(-\frac{i}{k}\right) \sum_{m=0}^{\infty}(2 m+1) b_{m} P_{m}(\cos \theta), k r \gg 1,
\end{aligned}
$$

where $m$ is the modal number, $b_{m}$ is the modal series coefficient, $P_{m}(\cos \theta)$ is the Legendre polynomial, $\theta$ is the (spherical) polar angle, and $h_{m}^{(1)}(k r)$ is the spherical Bessel function of the third kind (Hankel function of the first kind) of order $m$. The far-field limit ( $k r \gg 1)$ was given using the limiting form of the Hankel function $h_{m}^{(1)}(k r) \rightarrow(1 / k r) e^{i \mid k r-(m+1) \pi / 2\}}$ (Ref. 29).

From the above equation, the scattering amplitude is

$$
f(\Omega)=\frac{-i}{k} \sum_{m=0}^{\infty}(2 m+1) b_{m} P_{m}(\cos \theta) .
$$

For the backscattering case, $\theta=\pi, \quad \cos \theta=-1$, $P_{m}(-1)=(-1)^{m}$, and the backscattering cross section is

$$
\sigma_{\mathrm{bs}}=\frac{1}{k^{2}}\left|\sum_{m=0}^{\infty}(2 m+1) b_{m}(-1)^{m}\right|^{2} .
$$

In order to evaluate the above equations, $b_{m}$ must be determined by solving the boundary conditions. For the case of the spherical shell, Goodman and Stern originally published the coefficients, but limited the case to the inner and outer fluids being identical. The solution, involving solving six independent equations with six unknowns, is in the form of a ratio of two $6 \times 6$ determinants using Cramer's rule. It has been shown that their solution can be generalized to the case of the inner and outer fluids being different by a simple modification of the two nonzero terms in the sixth column of each determinant (replace $y$ with $y_{I I I}$ in both $\alpha_{46}$ and $\alpha_{56}$ and replace $\rho_{I}$ with $\rho_{I I I}$ in $\left.\alpha_{46}\right){ }^{4,5}$ The calculations in the numerical section of this article will involve use of the generalized determinants and the above equation for backscattering cross section. 


\section{B. Elongated objects-approximate solution}

While the solution in the previous section to the scattering by spherical objects was exact, the following deformed cylinder solution we use for the elongated objects is approximate. It will be applied to calculations of the scattering by prolate spheroids, straight finite cylinders, and uniformly bent finite cylinders. As discussed in the Introduction, we use the deformed cylinder solution because of its computational speed and accuracy at very high aspect ratios. In addition, it can be used to produce useful analytical solutions under limiting conditions.

The following assumptions are made in the general solution. (1) There is no dispersion or nonlinearities in the cylinder or surrounding medium. (2) End effects of the scattering by a finite cylinder are not important. This assumption restricts the solution to geometries where the directions of incidence and scattering are normal or near normal to the tangent of the axis of the cylinder. (3) Deformations of the cylinder axis, cross-sectional radius, and composition profile vary slowly with respect to position along the lengthwise axis of the cylinder. This comes from the part of the derivation that assumes the motion of neighboring cross sections of the elongated object to be similar allowing one to use the modal series coefficients that are derived from the infinitely long cylinder. It is this assumption that makes the calculations most accurate at high aspect ratios.

The general deformed cylinder solution, as derived in Ref. 24 , is

$$
p_{\mathrm{scar}} \cong \frac{-i}{\pi} P_{0} \int_{\mathrm{r}_{\mathrm{mss}}} \sum_{m=0}^{\infty} b_{m} \cos m \phi \frac{e^{i k\left(r_{\mathrm{s}}+\epsilon_{s}\right)}}{r_{s}}\left|\mathrm{~d} \mathbf{r}_{\mathrm{pos}}\right|,
$$

where $b_{m}$ is the modal series coefficient, $m$ is the modal number, $\phi$ is the azimuthal angle, $\mathbf{r}_{\text {pos }}$ is the position vector of the axis of the deformed cylinder, $r_{s}$ is the distance from the axis at point $\mathbf{r}_{\text {pos }}$ to the receiver, $\epsilon_{s}$ is the incremental or decremental distance between the point on the axis and the plane that both contains the origin and is perpendicular to the direction of the incident plane wave, and $\left|d \mathbf{r}_{\text {pos }}\right|$ is the differential of arc length. The details and geometry involving these terms are given in Ref. 24.

While this equation covers a broad range of geometries (i.e., both close and far from the object), it is desirable to examine it under the conditions where it will be used the most: at distances far from the object. Specifically, the receiver is far from the object so that $r \gg D_{e}$ and $D_{e} \ll 2 \sqrt{r \lambda}$, where $D_{e}$ is the distance between the two ends of the object and $2 \sqrt{r \lambda}$ is the diameter of the first Fresnel zone of the plane-wave source/point-receiver combination. This simplifies the formula so it reduces to a convenient form resulting in the following scattering amplitude:

$$
f(\Omega)=\frac{-i}{\pi} \int_{r_{\text {rax }}} \sum_{m=0}^{\infty} b_{m} \cos m \phi e^{i k r_{\text {pos }}\left(\hat{r}_{i}-\hat{r}_{r}\right) \cdot \hat{r}_{m \times x}}\left|d r_{\text {pos }}\right|,
$$

where $\hat{r}_{i}$ and $\hat{r}_{r}$ are the unit vectors in the direction of the incident plane-wave and scattered fields, respectively.

In the backscatter direction, $\phi=\pi$ and the backscattering cross section is

$$
\sigma_{\mathrm{bs}}=\frac{1}{\pi^{2}}\left|\int_{\mathbf{r}_{\mathrm{pos}}} \sum_{m=0}^{\infty} b_{m}(-1)^{m} e^{i k r_{\text {pos }}\left(\hat{r}_{i}-\hat{r}_{,}\right) \cdot \hat{r}_{\mathrm{pos}}}\right| d \mathbf{r}_{\mathrm{pos}}||^{2} .
$$

In order to solve the above equations, the geometry of the deformed cylinder and boundary conditions must be known. In each of the following three examples (prolate spheroid, straight finite cylinder, and uniformly bent finite cylinder), we show how the shape of the object is taken into account. For more details regarding the geometry, see the original derivations in Ref. 24 . In all three cases, the boundary conditions from the infinitely long cylinder (assumption 3 ) are used. For the case of elastic shelled objects, which is the focus of this article, we use the coefficients initially published by Doolittle and Überall ${ }^{7}$ and later in normalized form by Uginčius and Überall ${ }^{8}$ for our elongated objects.

\section{Straight finite cylinder}

For a straight cylinder of length $L$, the modal series in the above equations comes out of the integral, and the scattering amplitude was shown in Refs. 22 and 24 to be

$$
f(\Omega)=\frac{-i L \sin \Delta}{\pi \Delta} \sum_{m=0}^{\infty} b_{m} \cos m \phi,
$$

where $\Delta=\frac{1}{2} k L\left(\hat{r}_{i}-\hat{r}_{r}\right) \cdot \hat{r}_{\tan }, \hat{r}_{\tan }$ is the unit vector in the direction of the tangent of the cylinder axis, and all wave numbers in $b_{m}$ are multiplied by $|\sin \theta|$ where $\theta$ equals the angle between the direction of the incident wave and the axis of the cylinder.

In the case of backscatter, $\phi=\pi$ and the backscattering cross section is

$$
\sigma_{\mathrm{bs}}=\frac{L^{2}}{\pi^{2}} \frac{\sin ^{2} \Delta}{\Delta^{2}}\left|\sum_{m=0}^{\infty} b_{m}(-1)^{m}\right|^{2},
$$

where now $\Delta=k L \cos \theta$.

\section{Prolate spheroid}

When using the deformed cylinder formulation to describe the scattering by a prolate spheroid, we must express the cylindrical radius in terms of the position along the axis. For simplicity, we place the center of the spheroid at the origin which gives the equation for the radius:

$$
a(z)=a_{0} \sqrt{1-[z /(L / 2)]^{2}},
$$

where $z$ is the distance along the major axis, the total length of the spheroid is $L$, and the length of its semiminor axis is $a_{0}$.

Making the substitution of $\mu=z /(L / 2)$ and $d \mu=d z /(L / 2)$ and taking advantage of the fact that the integral is of an even function over a symmetrical interval about the origin, the backscattering cross section at broadside incidence is ${ }^{24}$

$$
\begin{aligned}
\sigma_{\mathrm{bs}} & =\frac{1}{4 \pi^{2}} L^{2}\left|\int_{-1}^{1} \sum_{m=0}^{\infty} b_{m}(-1)^{m} d \mu\right|^{2} \\
& =\frac{1}{\pi^{2}} L^{2}\left|\int_{0}^{1} \sum_{m=0}^{\infty} b_{m}(-1)^{m} d \mu\right|^{2},
\end{aligned}
$$

where now $a(\mu)=a_{0} \sqrt{1-\mu^{2}}$ and $\phi$ was set equal to $\pi$. Because we have broadside incidence, the exponent in the integrand in the general deformed cylinder solution is zero and the wave numbers in $b_{m}$ remain unmodified. 


\section{Uniform/y bent finite cylinder}

The bend of the uniformly bent cylinder is taken into account both in the exponent in the integrand in the deformed cylinder solution and in the wave numbers: ${ }^{24}$

$$
\begin{aligned}
\sigma_{\mathrm{bs}} & =\frac{\rho_{c}^{2}}{\pi^{2}}\left|\int_{-\gamma_{\max }}^{\gamma_{\max }} \sum_{m=0}^{\infty} b_{m}(-1)^{m} e^{2 i k \rho_{c}(1-\cos \gamma)} d \gamma\right|^{2} \\
& =\frac{4 \rho_{c}^{2}}{\pi^{2}}\left|\int_{0}^{\gamma_{\max }} \sum_{m=0}^{\infty} b_{m}(-1)^{m} e^{2 i k \rho_{c}(1-\cos \gamma)} d \gamma\right|^{2}
\end{aligned}
$$

where $\rho_{c}$ is the radius of curvature of the axis of the cylinder, the angle $2 \gamma_{\max }$ subtends the entire arc, the total arc length $L=2 p_{c} \gamma_{\max }$, and $\phi=\pi$ for all $\gamma$. All wave numbers in $b_{m}$ are multiplied by $|\cos \gamma|$.

The geometry of this case is shown simplistically in the figures in the next section and in detail in Ref. 24. The cylinder is bent symmetrically away from the sonar.

\section{NUMERICAL CALCULATIONS}

In this section, we present numerical calculations illustrating the backscattering characteristics of the following shelled objects: sphere, prolate spheroid, straight finite cylinder, and uniformly bent finite cylinder with the latter three (elongated) objects having high aspect ratios ( $\gtrsim 5$ ). As discussed in Sec. I, the calculations are exact for the spheres and approximate for the elongated objects. The calculations were carried out for broadside incidence for each of the elongated objects as that is the geometry where the deformed cylinder solution is the most accurate (the scattering geometry is illustrated for each figure). The shell of each object is composed of stainless steel and the surrounding fluid is water (the material properties were taken from Neubauer ${ }^{30}$ ). The scattering predictions were performed for a wide range of $k a$ and shell thicknesses for several interiors: (1) water with identical properties as surrounding water, (2) air, and (3) fluid with similar but not identical properties as surrounding water. The last interior material was chosen as the acoustic properties of some marine organisms such as shrimp and euphausiids are close to that of water. ${ }^{31}$ Note that the set of plots that would correspond to Fig. 1 for the dissimilar fluid interior [interior (3)] is not shown because the results were so close to those of Fig. 1. The differences in the calculations show up in Figs. 2 and 3, where the shell is very thin.

The computer algorithms were based on the equations summarized in Sec. I of this article. There were three classes of modal series coefficients used. (1) Fluid-solid for the top row of Figs. 1 and 4 and all of Fig. 6. All of these calculations used the coefficients derived by Faran. ${ }^{32}$ Faran's sphere coefficients were used for the solid sphere and his cylinder coefficients for the solid prolate spheroid, straight finite cylinder, and uniformly bent cylinder. (2) Fluid-solid-fluid for the calculations involving the shell. As described in Sec. I, the coefficients for the sphere were originally derived by Goodman and Stern ${ }^{1}$ and later generalized by Poggio. ${ }^{5}$ The cylinder coefficients that were used for all elongated objects were originally derived in Doolittle and Überall ${ }^{7}$ and published in normalized form by Uginčius and Überall. ${ }^{8}$ (3) Fluid-fluid for the bottom row of Figs. 3 and 5. The coefficients for the fluid sphere were originally derived in Anderson, ${ }^{33}$ while the coefficients for the fluid cylinder that were used for all elongated objects were taken from Stanton. ${ }^{22}$

Figures 1-5 summarize our calculations. The "reduced" or normalized target strength is plotted as a function of $k a$ for each object. The direction of the incident wave is indicated by the arrow over each object. The terms reduced and " $a$ " have the same meaning as in Ref. 24: The target strength is reduced by $10 \log \pi a^{2}$ for the spheres and $10 \log L^{2}$ for each elongated object where $a$ is the radius of the spheres and $L$ is the length of the prolate spheroid and straight cylinders and the arc length of the bent cylinders. The reduction is made so that the argument of the logarithm for the vertical axis may be dimensionless. The $a$ in the horizontal axis term $k a$ is the (spherical) radius of the spheres, (cylindrical) radius of the straight and uniformly bent cylinders, and length of the semiminor axis of the prolate spheroids. The shell cross section is shown on the left-hand side of each row of the plots. The term " $\mathrm{ft}$ " indicates the fractional thickness of each shell and in all cases but the prolate spheroid is equal to the ratio of the shell thickness to the outer radius $a$. In those cases, the fractional shell thickness can be expressed in terms of $a$ and the inner radius $b$ as $\mathrm{ft}=1-b / a$. In the case of the prolate spheroid, the radial component of the thickness of the shell is held constant along the length of the object and $\mathrm{ft}$ is equal to the ratio of the shell thickness at the origin to the length of the outer semiminor axis. The inner walls of the prolate spheroid eventually meet near the ends (the point at which they meet depends, of course, on the shell thickness) and the computer program took this into account by calculating the scattering due to a shell with a negligibly small interior beyond this point (i.e., the ends were effectively solid). Since the calculations involve high aspect objects, the portion of the spheroid near the ends that deviates significantly from being of uniform thickness is small; hence the spheroid can be considered, to a reasonable approximation, of uniform thickness. (In order to illustrate detail of the resonance structure in the abovementioned plots, some of the curves in Figs. 1 and 4 are replotted on linear scales in Figs. 6-8.)

All plots are characterized by a Rayleigh scattering region in the $k a \ll 1$ region where the backscattering cross section varies at $(k a)^{4}$. In the $k a>1$ region, all plots approach the geometric scattering limit. The slope of the limit varies from object to object and is discussed in detail in Ref. 24. In general, the slope is zero for the sphere, prolate spheroid, and bent cylinder and nonzero (positive) for the straight cylinder. These differences in slopes are due to Fresnel zone effects that depend on the fact that the sphere, spheroid, and bent cylinder are curved in two dimensions, while the straight cylinder is curved only in one dimension. The plots show that for shell fractional thicknesses greater than about 0.5 , the cross sections are relatively independent of the inner material. This is because the density and speed of sound of stainless steel are greater than those corresponding properties of the surrounding water and hence the shell is relatively impenetrable for those thicknesses.

As the shell becomes thinner, the effect of the interior becomes apparent. In the $k a>1$ region, the modal interfer- 


\section{REDUCED TARGET STRENGTH VS ka}
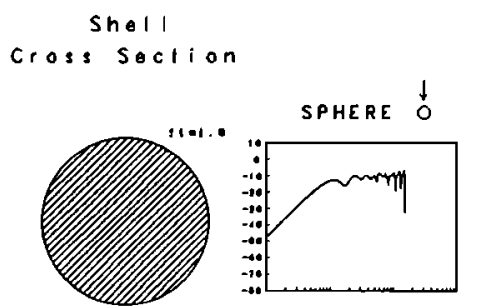

PROLATE
SPHEROID

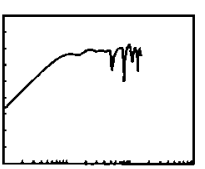

STRAIGH CYLINDER
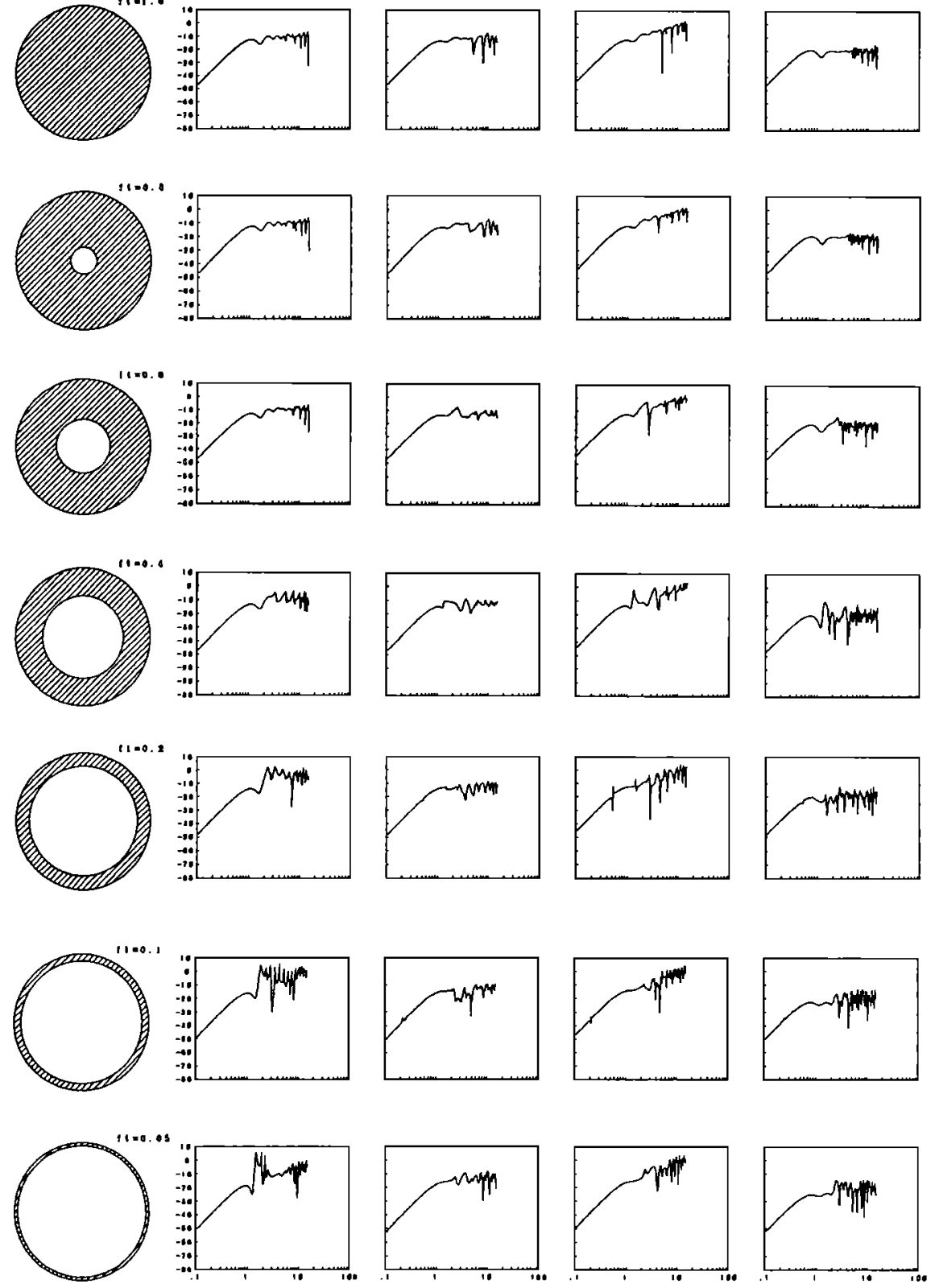

k a

FIG. 1. Reduced (normalized) target strength versus ka for the sphere, prolate spheroid, straight finite-length cylinder, and uniformly bent finite cylinder for various shell thicknesses. The plots show the trend and resonance structure of the backscattering cross section to depend strongly on body shape and shell thickness in the $k a>1$ (geometric) region. All plots are characterized by a smooth monotonically increasing curve in the $k a \ll 1$ (Rayleigh) region. The elastic shell of each object is constructed of stainless steel while the inner and outer fluids are both water. The reduced target strength for the corresponding solid elastic objects (no inner fluid) is given on the top row for comparison. Direction of incident plane wave is given by arrow. All calculations involving the sphere are exact as they involve direct use of the Faran ${ }^{32}$ (solid) or Goodman and Stern' (shell) sphere formulations. The calculations involving all elongated objects are approximate as they involve use of the Faran ${ }^{32}$ (solid) and Uginčius and Überall ${ }^{8}$ (shell) cylinder coefficients in the deformed cylinder formulation described in this article. Because of the use of the cylinder coefficients that were derived for infinitely long and straight cylinders, the predictions are accurate only for high aspect ratios (ratio of length to diameter greater than about 5 ). ${ }^{24}$ The following material properties were used: water, $c_{w}=1496.59$ $\mathrm{m} / \mathrm{s}$ (tap water at $25^{\circ} \mathrm{C}$ ); stainless steel, specific density $g=7.90, c_{c}=5594 \mathrm{~m} / \mathrm{s}$, and $c_{s}=3106 \mathrm{~m} / \mathrm{s}$, where $c_{c}$ and $c_{s}$ are the compressional and shear speeds of sound, respectively. ${ }^{30}$ The resultant ratios of speeds of sound used in the programs were then $h_{c} \equiv c_{c} / c_{w}=3.74$ and $h_{s} \equiv c_{s} / c_{w}=2.08$. For the calculations involving the bent cylinder, the ratios $\rho_{c} / L=0.5$ and $L / a=10.5$ were used (these were values consistent with the shape of certain marine organisms).$^{24}$ All modal series in the solutions are converged using the first 20 modes $(0 \leqslant m \leqslant 19)$. 


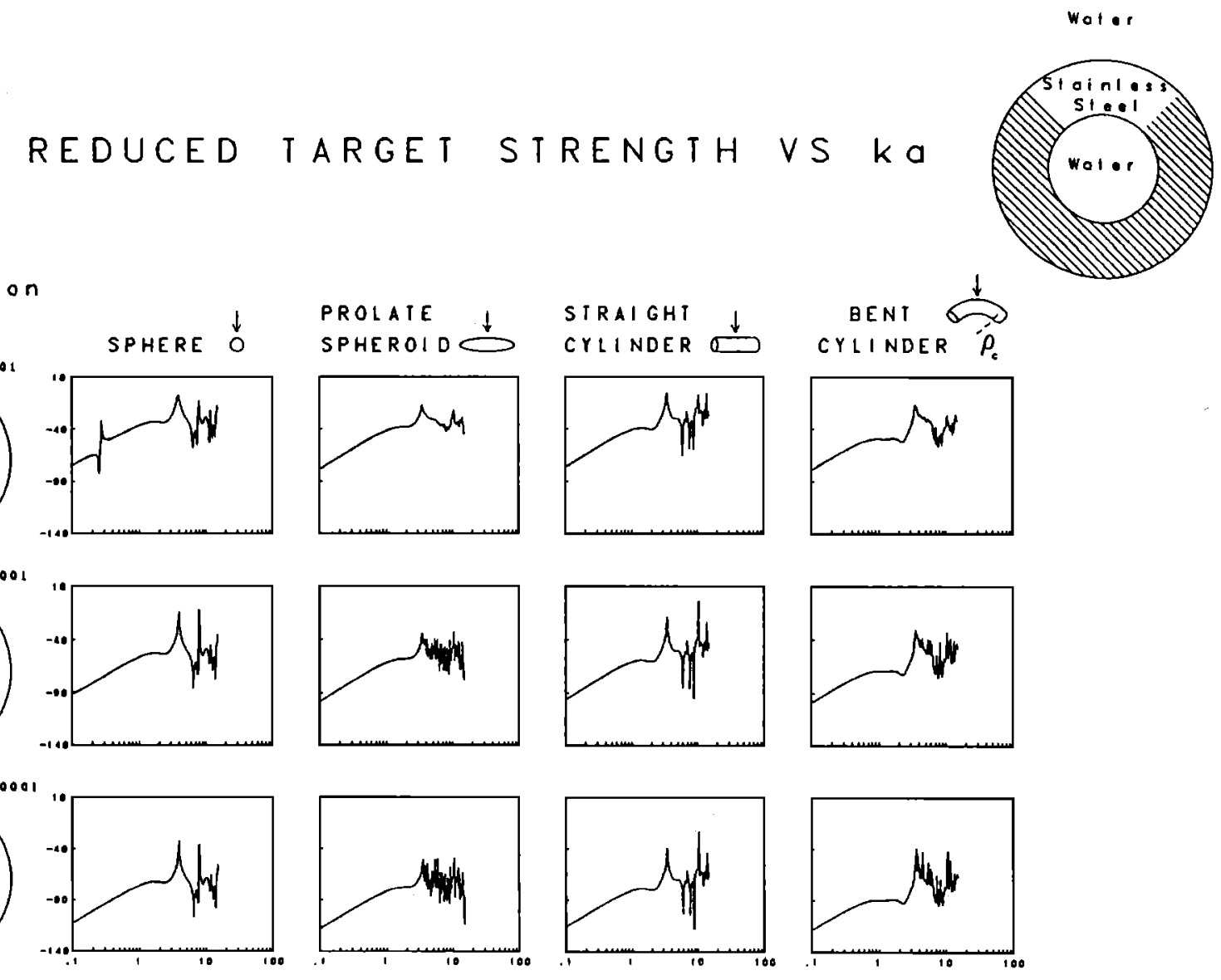

$\mathrm{ka}$

FIG. 2. Continuation of Fig. 1 for very small shell thicknesses. This set of plots is on a different vertical scale than in Fig. 1 to illustrate the great reduction in overall levels as the thickness of the shell is reduced.

ence structure changes dramatically. This is due to both variations in the internal reflections within the interior and shell as well as changes in the surface elastic waves (such as the introduction of Lamb waves). ${ }^{34}$ For fluid interiors (water and "fluid"), the backscattering cross section decreases with decreasing shell thickness in the $k a \ll 1$ region. This is due to the fact that $k \delta \ll 1$ also in this region, where $\delta$ is the thickness of the shell, thus the thickness of the shell is also in the Rayleigh scattering region. For thin spherical shells where the inner fluid is identical to the outer fluid, the cross section can be shown to vary as $\sigma_{\text {bs }} \sim \delta^{2}$ (this is true for any $k a$ provided $k \delta \ll 1) .^{1}$ The plots from all shapes where the inner fluid is identical to the outer fluid show this behavior for sufficiently thin shells. At $k a=0.1$, the relation $\sigma_{\mathrm{bs}} \sim \delta^{2}$ was correct to within $0.1 \mathrm{~dB}$ below values of $\mathrm{ft}$ of about $10^{-4}$ for the elongated objects and $10^{-5}$ for the sphere (these results not shown). At higher $k a$ values, the curves also appear to have similar behavior although there are variations due to modal interferences at the highest $k a$ values. When the inner fluid is different than the surrounding water (Fig. 3) the plots decrease until the point at which they converge to the no-shell or fluid-only solution (no-shell calculations using fluid-fluid boundary conditions).

The shape of the plots for the air-filled shells changes quite dramatically as the thickness of the shell is decreased.
As the effect of the shell decreases, a resonance in the $k a \ll 1$ region appears and shifts toward lower $k a$ values. Figure 5 shows the solution to approach the no-shell or gas object solution (no-shell calculations using fluid-fluid conditions). In contrast to the cases when the shells had denser interiors (water and "fluid") the level and trend of the curves in the geometric scattering regions in the thin gasfilled case are similar to those in the solid case. This is because once the effects of the shell are negligible, the nearly impenetrable air will give a similar return as the nearly impenetrable solid stainless steel.

\section{EXPERIMENT}

Because of the limited amount of data available in the literature involving scattering of sound by elastic shelled objects (especially finite sized ones), we have begun a series of measurements to further investigate the scattering properties of such objects. The author is not aware of any data involving scattering by uniformly bent finite cylindrical shelled objects and, since that particular shape has shown to be important in describing the scattering by marine organisms, ${ }^{24}$ our first measurements have involved that object as well as a straight finite cylindrical shell for comparison. The measurements involved normal incidence backscatter, pings sufficiently long so that individual scattering highlights co- 


\section{REDUCED TARGET STRENGTH VS Ka}
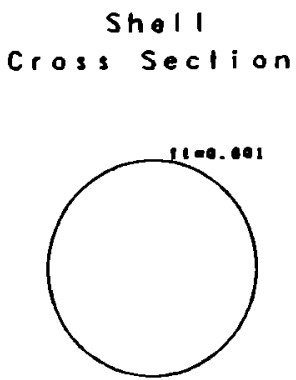
SPHERE $\stackrel{\downarrow}{0}$
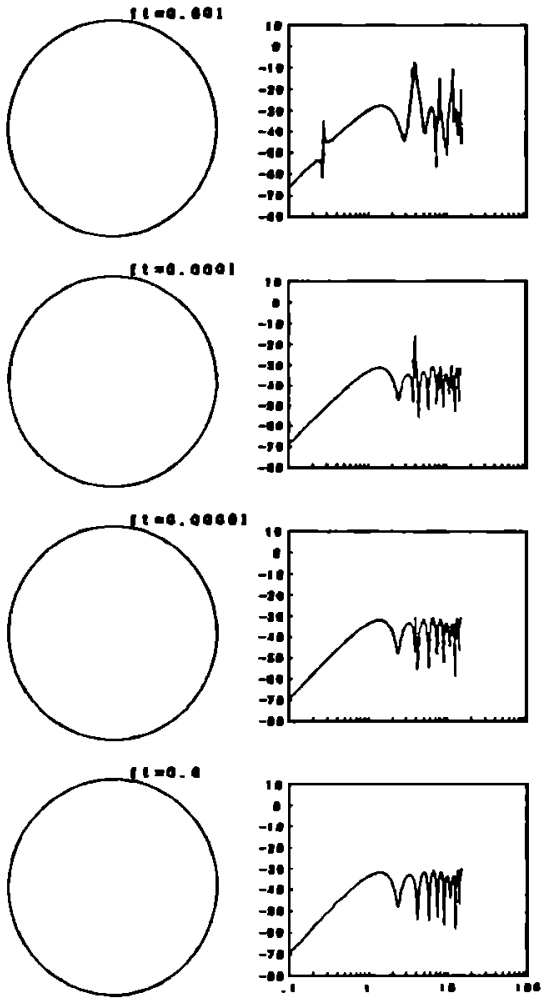

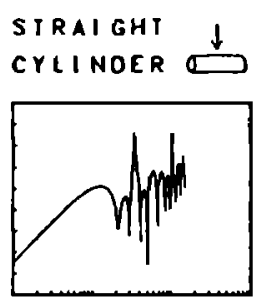

BENT
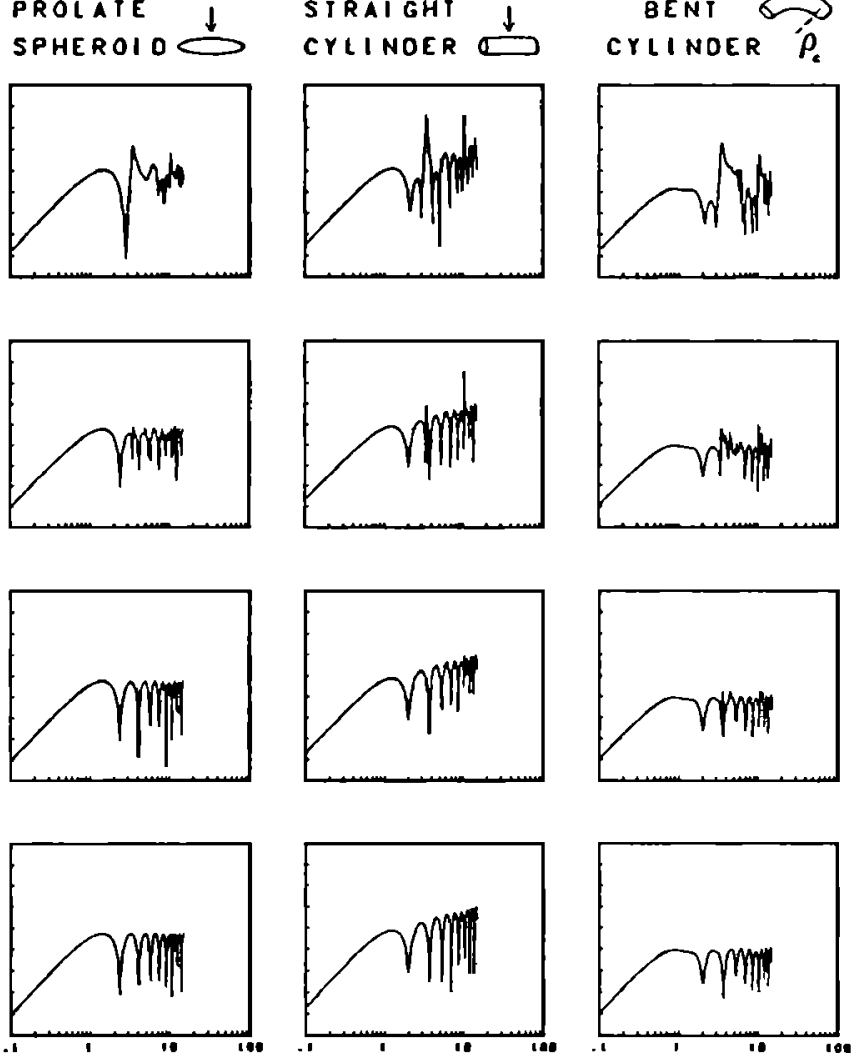

k o

FIG. 3. Backscattering predictions with an interior fluid slightly different than the exterior fluid. The interior fluid is chosen to have properties similar to those of marine organisms: specific density $g=1.043$ and specific speed of sound $h\left(\equiv h_{c}\right)=1.052 .{ }^{24}$ The figure shows the predicted values to converge to the no-shell calculations given on the bottom row. Note that, although the shell thicknesses are the same as in Fig. 2, these plots are on the same scale as in Fig. 1 . The stainless steel parameters and shape parameters are given in the caption to Fig. 1 .

incided (the long ping mode is convenient for modal series analysis), and at frequencies and sizes such that $3<k a<9$.

\section{A. Experimental setup}

Our measurements were conducted in a 4-foot diameter by 3-foot high circular tank at the Woods Hole Oceanographic Institution. Because of the small size of the tank, our measurements were limited to small objects (approximately $0.5 \mathrm{~cm}$ in length) and ultrasonic frequencies (1-2.5 MHz). In order to examine the structure of the backscattering cross section curve versus $k a$, we used broadband transducers similar to those used in medical applications to provide nearly continuous coverage (closely spaced discrete frequencies) over the entire frequency range. Two closely spaced transducers were used, one for transmission and one for reception to obtain the backscatter data. This approach was used in- stead of a single transducer in order to increase signal to noise ratio and to make calibration more accurate. Calculations show that there is a negligible difference between a true monostatic (single transducer) experiment and a bistatic experiment (two transducers) where the transducer pair subtends a 2-deg angle such as ours.

Commercially available pulse-echo equipment was used for the measurements. A gated sine wave was applied to the transmission transducer by use of a Hewlett-Packard 8011 A pulse generator (gate), Hewlett-Packard 8116A pulse/ function generator (sine wave modulated by the gate), and ENI $2100 \mathrm{~L}$ power amplifier. There was no need for an impedance matching circuit between the power amplifier and the transmission transducer as the input impedance of the transducer was close to the 50-ohm impedance of the amplifier. The two identical transducers were (custom) con- 
Shel 1

cross section SPHERE
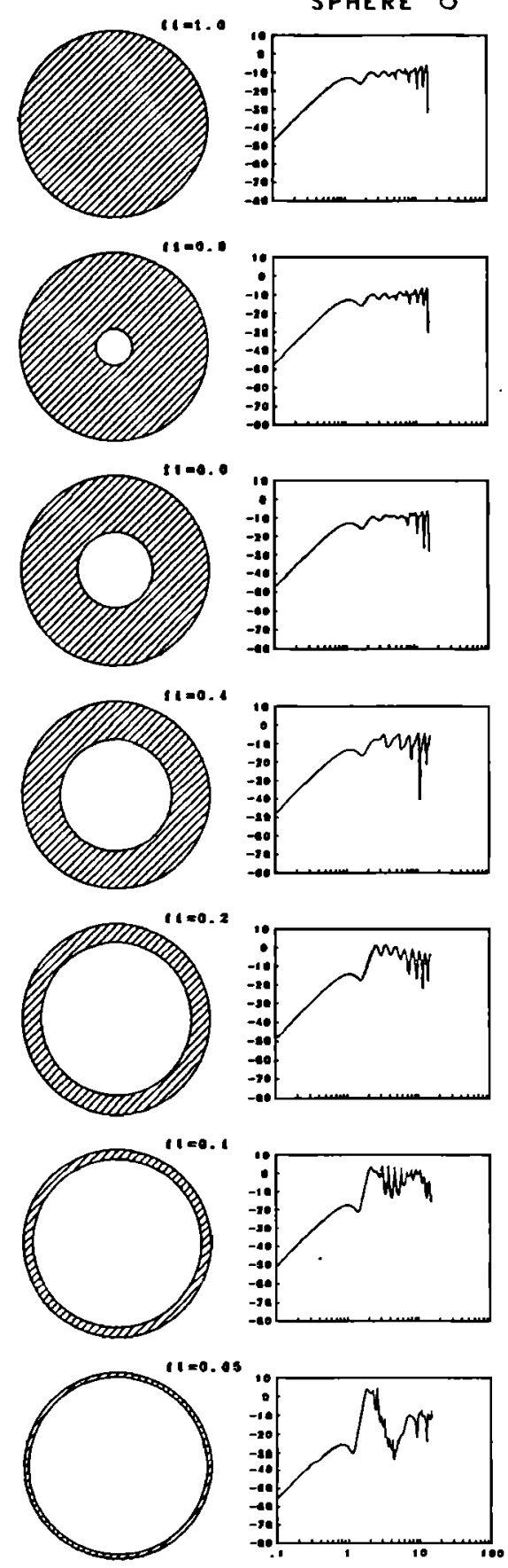
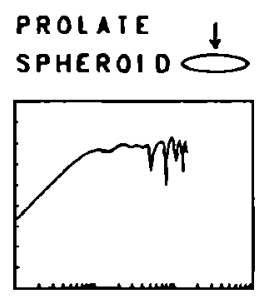

STRAIGHT
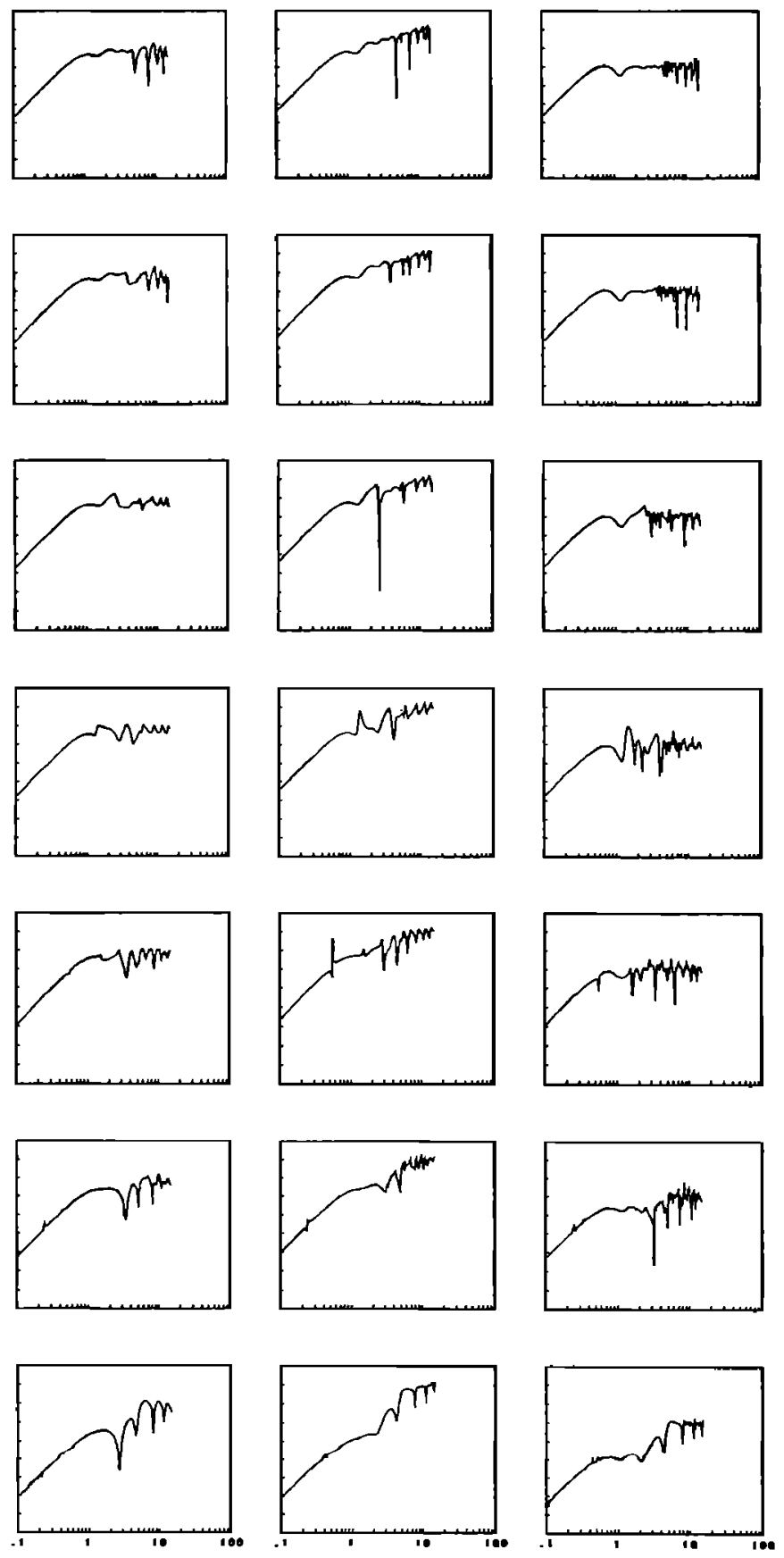

k a

FIG. 4. Backscattering predictions for air-filled objects. Since air is considered a fluid in these calculations (no shear waves), the same programs used to calculate the plots in Fig. 1 were used here. All parameters were the same with the exception of the interior parameters: specific density $g=0.0012$ and specific speed of sound $h=0.22$ (1-atm air). 


\section{REDUCED TARGET STRENGTH VS Ka}

Shel 1

cross section
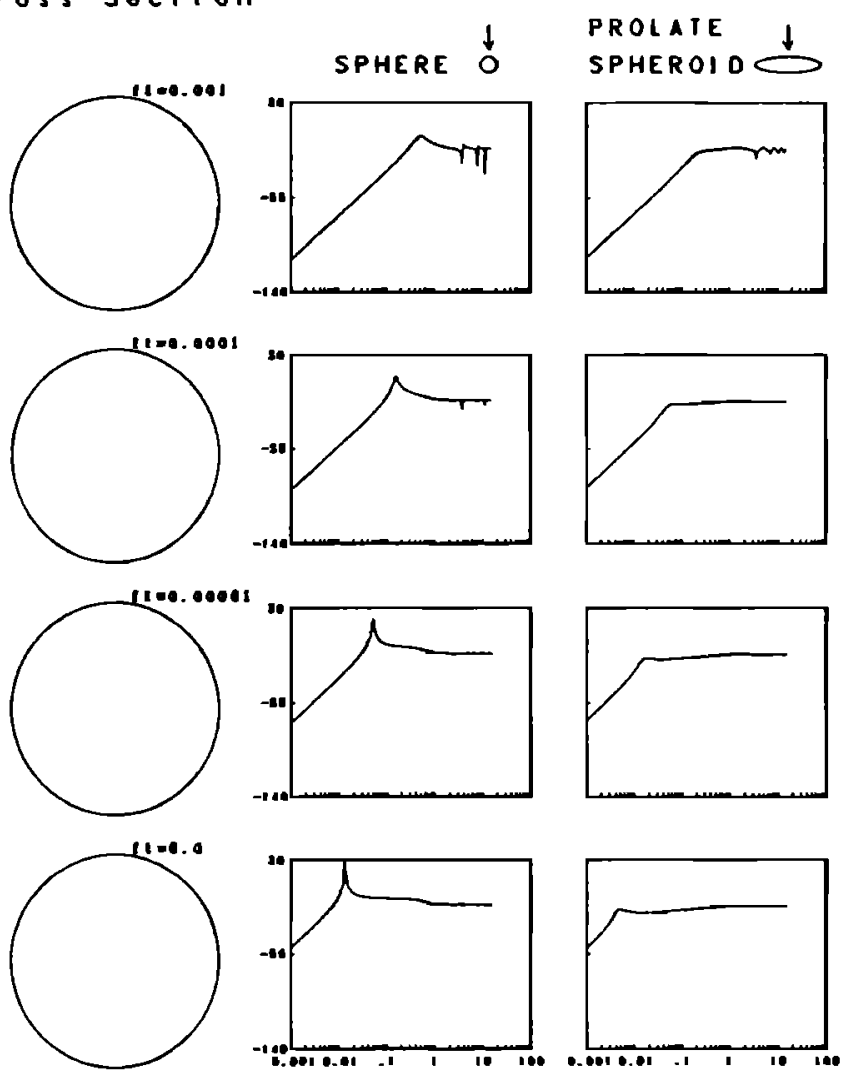
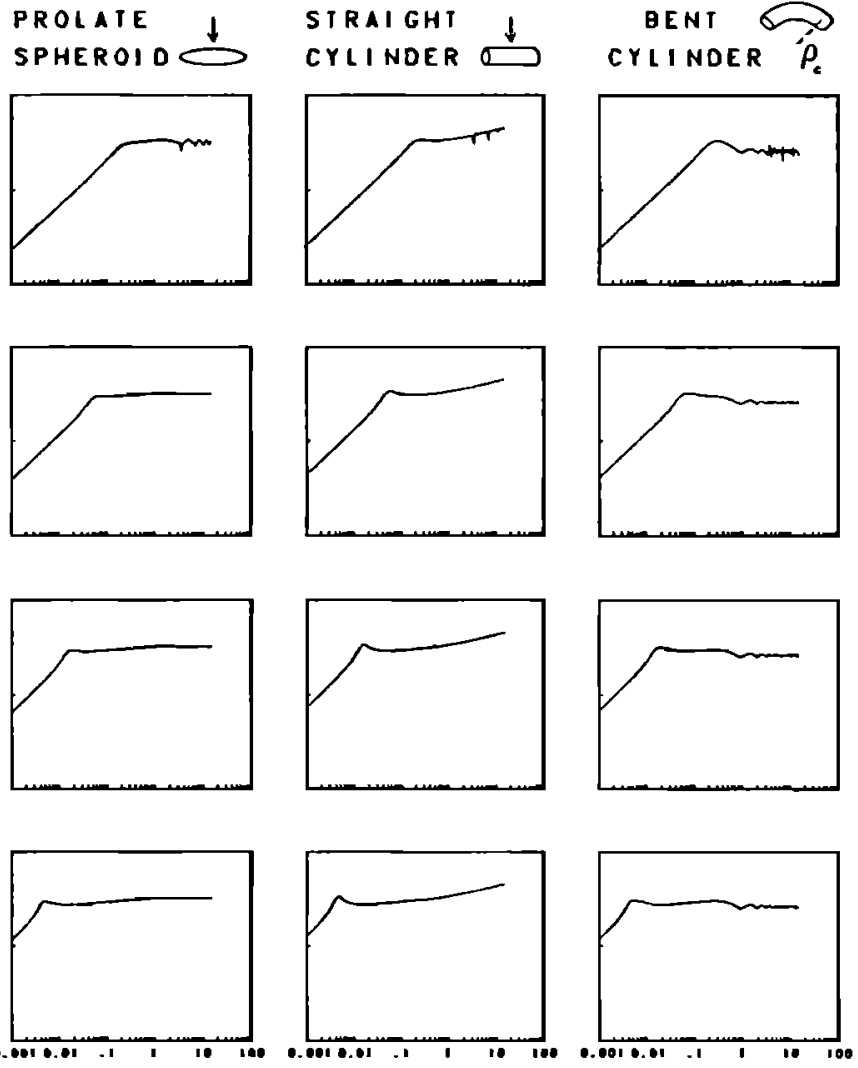

k a

FIG. 5. Continuation of Fig. 4 for very small shell thicknesses. This set of plots is on a different scale to illustrate the evolution of the resonance due to the interior. The curves are shown to approach the no-shell calculations given on the bottom row.

structed by Krautkramer Branson. Each transducer contained a 1-in.-diam ceramic with a center frequency of 2.25 MHz. The ceramic and backing materials were chosen so that the ceramic would be heavily damped and broadband excitation would be possible ("Alpha Series, Broadband IS Style"). For our purposes, the transducers had a usable frequency range of approximately $1-2.5 \mathrm{MHz}$. The cable was potted so that the entire unit could be immersed in water. Because of the high frequencies and short wavelengths involved, use of a flat ceramic would have produced unacceptably narrow beamwidths as it would have been difficult to position the objects in the center of the beam. We therefore used ceramics in the shape of spherical caps to provide 20deg beamwidths.

The echoes detected by the receiving transducer were amplified, filtered, and finally displayed on an oscilloscope. The battery powered amplifier (preamp) was a modified version of model 5662 made by Panametrics. The modifications (done by Panametrics) extended the upper limit of the frequency range to $5 \mathrm{MHz}$ so that the entire range is now 10 kHz-5 MHz. The filter, model AP220-5-R from AP Circuit, was used as a high-pass filter with the cutoff below our frequency range. It could not be used in the bandpass mode in our frequency range without reducing the level of the upper frequencies. The Tektronix oscilloscope, model 2445B, both displayed the echoes and was used in determining the amplitudes of the echoes. A manually adjustable line cursor on the scope was adjusted until its level coincided with the peak of the echo. A digital display of the level was then manually recorded.

The shelled objects were constructed of stainless steel tubes purchased from Alltech Associates, Inc. The tubes, catalog No. 97095, were designed for gas chromatographic applications and were originally $5 \mathrm{~cm}$ long by $1 / 16$-in. out- 
$F T=1.0$

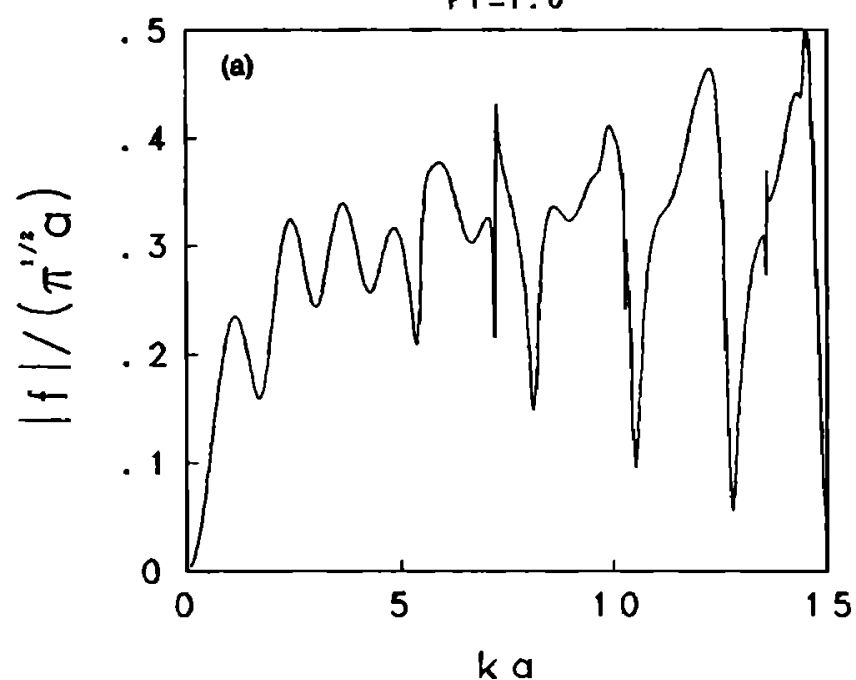

PROLATE SPHEROID

SOLIO STAINLESS STEEL

F $T=1.0$

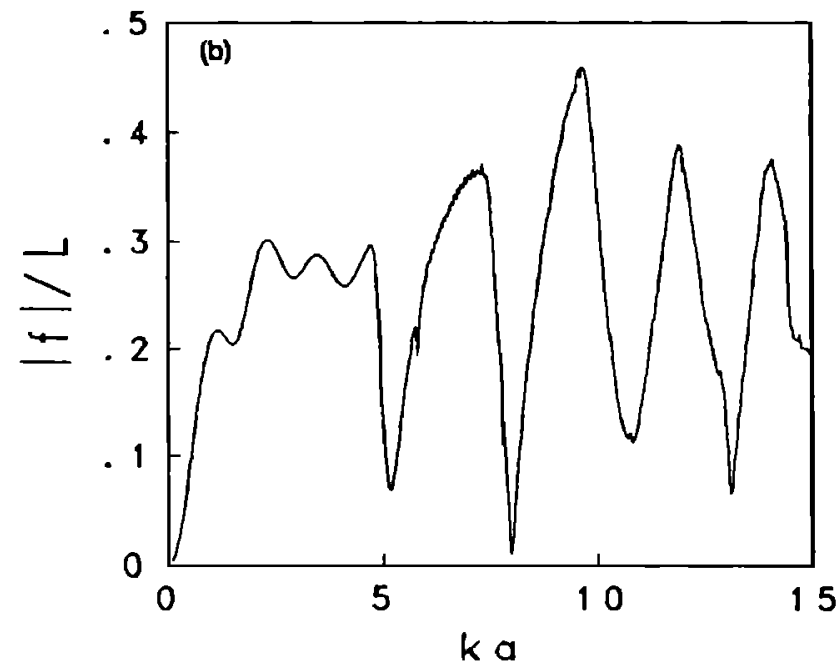

FIG. 6. Expanded plots from top row of Fig. 1 on linear scales.

side diameter with a 0.03 -in. inside diameter. The ratio of the shell wall thickness to the outer radius of each tube (distance between center and outer boundary) is 0.52 . This corresponds to a ratio of $b / a=0.48$, where $b$ is the inner radius. Construction of the straight tube involved carefully cutting the tube so as not to deform it. This required use of a tube cutter made specifically for these 1/16-in.-diam tubes (cata$\log$ No. 3165, also made by Alttech). We attempted to cut a 5-mm section of straight tube and photocopy blowups of the tube show the actual length to be $4.8 \mathrm{~mm}$. Construction of the bent tube was more complicated as we needed to first bend the long section, then cut it. It was difficult to obtain a uniformly bent tube for small radii of curvature. After con-

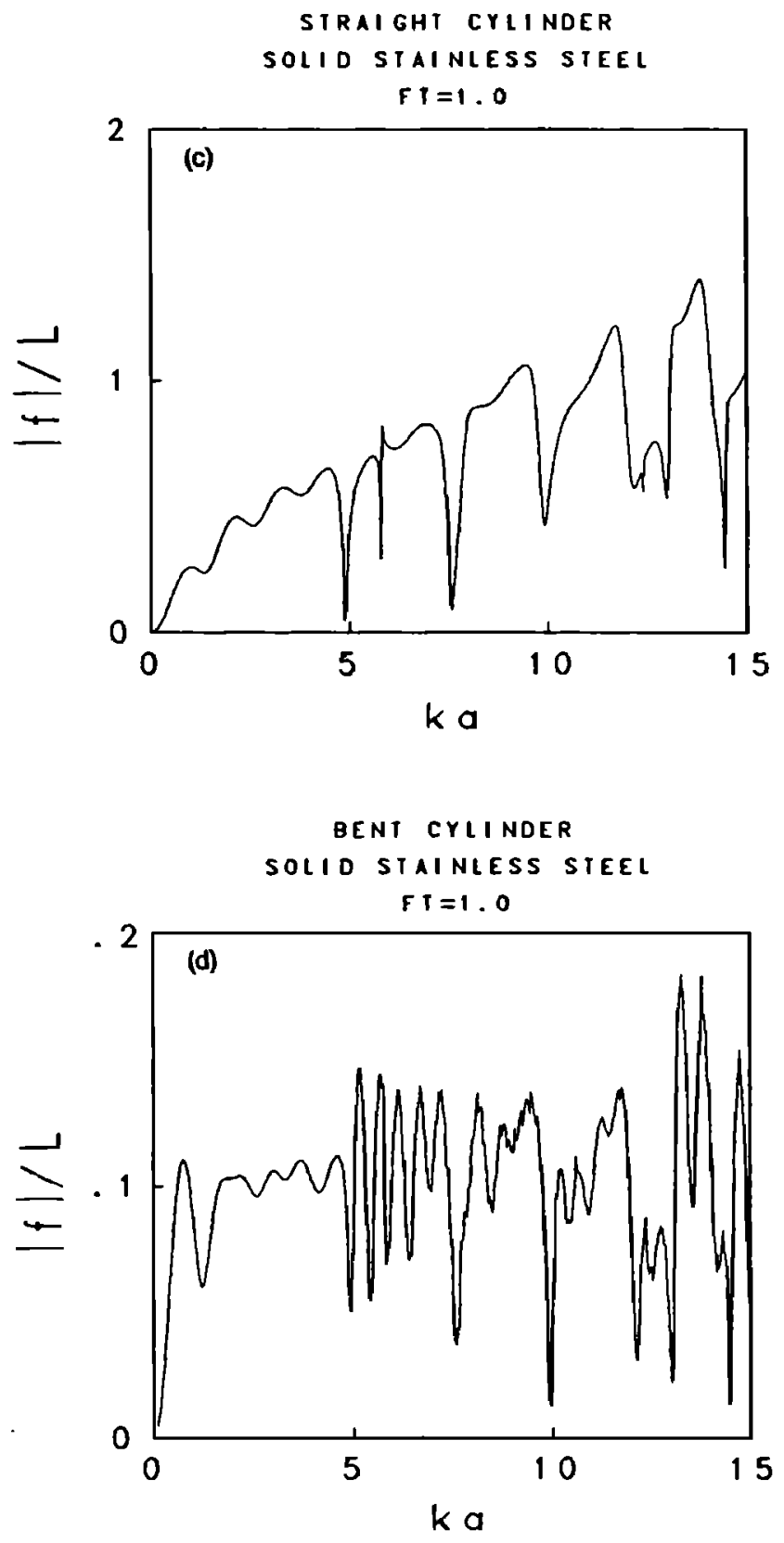

structing several bent tubes, we ended up with one that was satisfactory with an arclength $L$ of $4.8 \mathrm{~mm}$ and radius of curvature $\rho_{c}$ of $6.0 \mathrm{~mm}$ giving a ratio $\rho_{c} / L=1.25$. The ratio $L / a$ for the bent tube was equal to 6.05 giving an aspect ratio $L / 2 a=3.02$.

The tubes were placed at distances of at least $75 \mathrm{~cm}$ from the transducers. At the highest frequency, $2.5 \mathrm{MHz}$, and at a 75-cm range, the diameter of the first Fresnel zone $(\sqrt{2 r \lambda})$ of the point-source/point-receiver combination is equal to $3.0 \mathrm{~cm}$. At greater ranges and/or lower frequencies, the Fresnel zone is larger than $3.0 \mathrm{~cm}$. With shell lengths of approximately $0.5 \mathrm{~cm}$, the shells are well within the first Fresnel zone and hence they are considered "finite" in size 
$\mathrm{F} \mathrm{T}=.05$

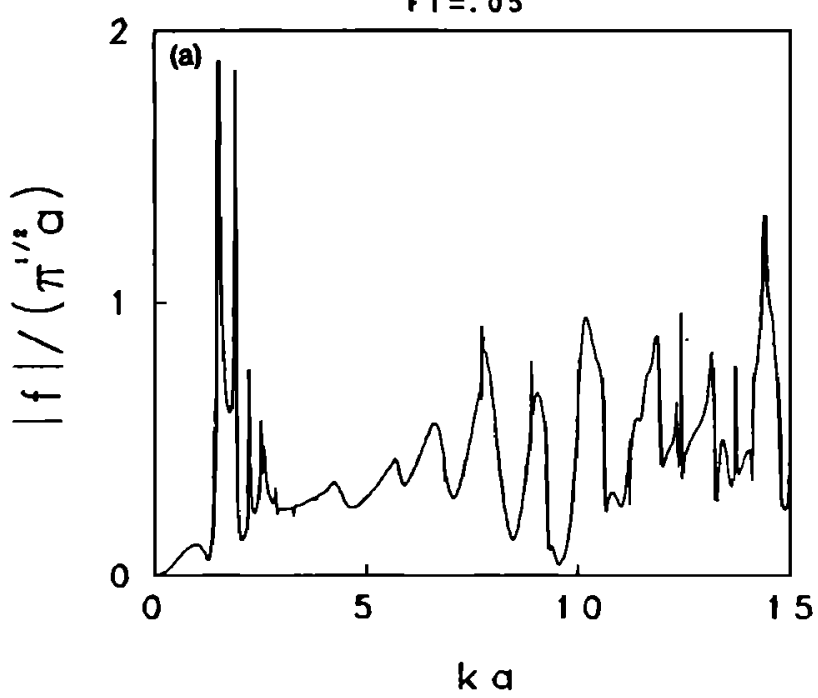

PROLATE SPHEROID

WATER-FILLED STAINLESS STEEL SHELL $F \mathbf{T}=.05$

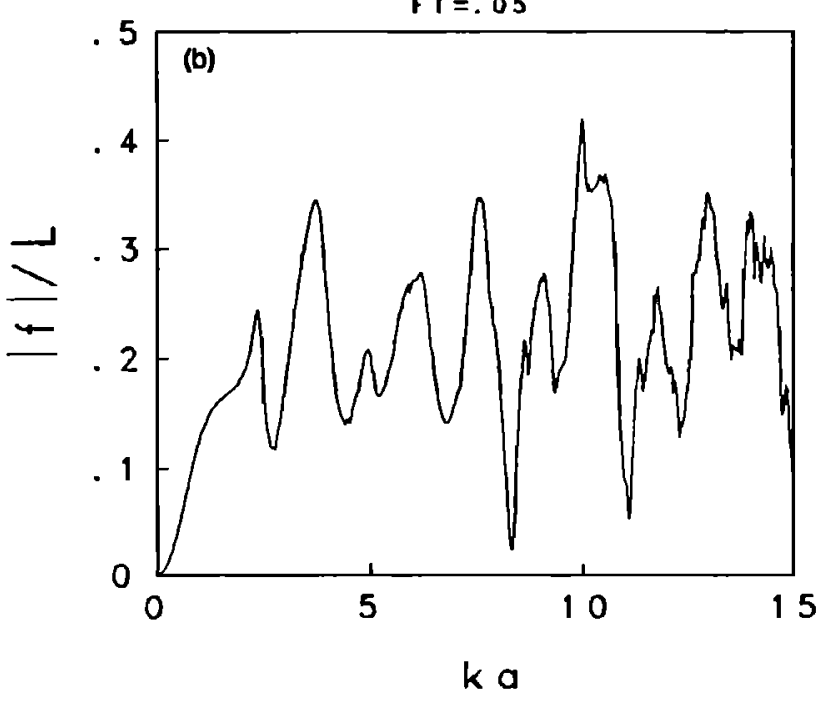

FIG. 7. Expanded plots from bottom row of Fig. 1 on linear scales.

with respect to this measurement. The finite deformed cylinder equations described in Sec. I of this article then are applicable to this measurement.

The major challenge in this experiment was the alignment of the objects and transducers. The transducers (mounted on test tube holders) were first aligned according to mechanical measurements so that they would share the same volume of insonification at a range of approximately 75 $\mathrm{cm}$. They were then individually operated in the monostatic mode while the fields at $75-\mathrm{cm}$ range were mapped with a suspended sphere (ball bearing). The study showed the transducers to be initially slightly misaligned and the orientations were then altered accordingly.

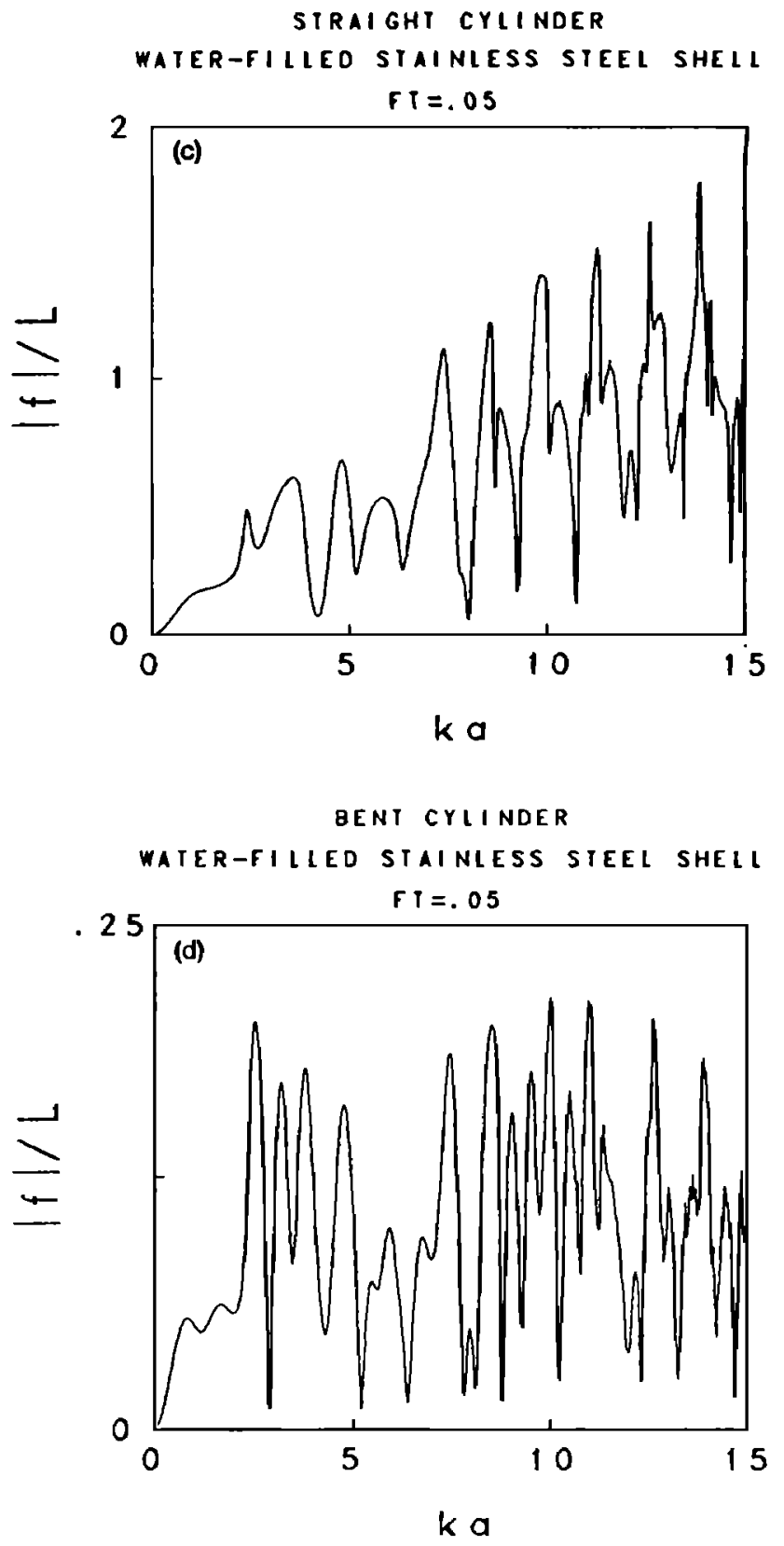

The tubes were suspended by long strands of snugly strung human hair. The hair turned out to be an excellent mounting material as it is acoustically transparent at these frequencies. By appropriate use of the hair and support rods well away from the acoustic beams, the echo from the $75-\mathrm{cm}$ range was solely from the tubes and not from any surrounding mount. One strand of hair, suspended between two vertical rods, was threaded through the tubes to allow control of the orientation of the tubes. An additional strand, used in the bent tube measurements, was strung from the top of the upper transducer, wrapped around the midsection of the tube, and then back to the bottom of the lower transducer. It was used to pull the midsection of the tube toward the trans- 


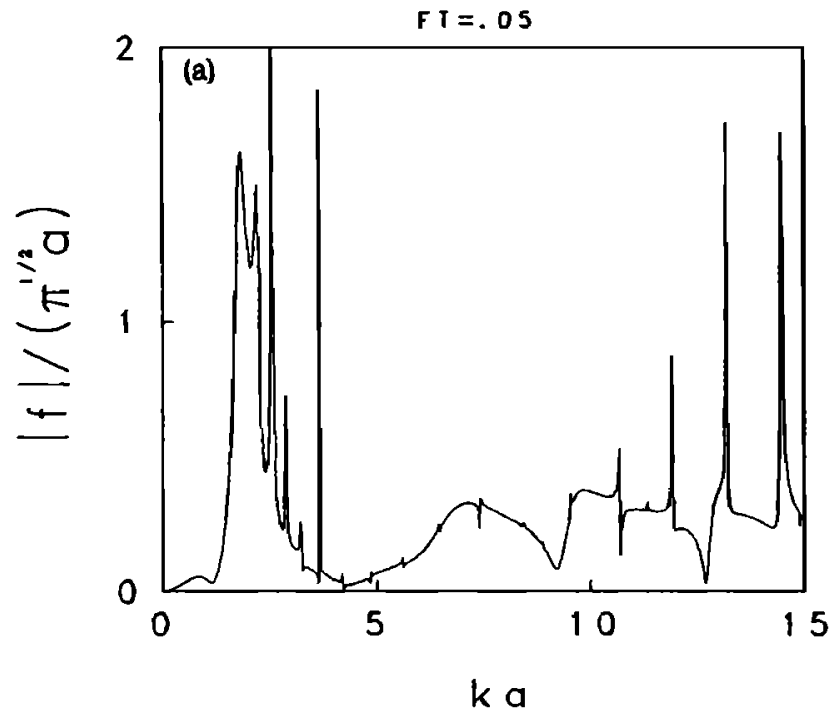

AIR-FILLED STAINLESS STEEL SHELL

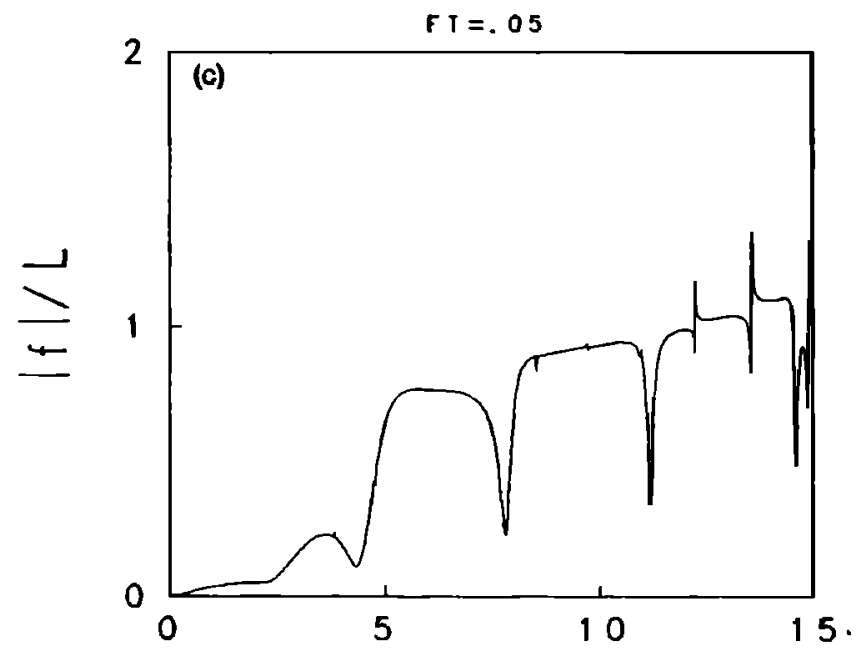

ka

PROLATE SPHEROID

AIR-FILLED STAINLESS STEEL SHELL

$F \mathrm{~T}=.0 \mathrm{~S}$
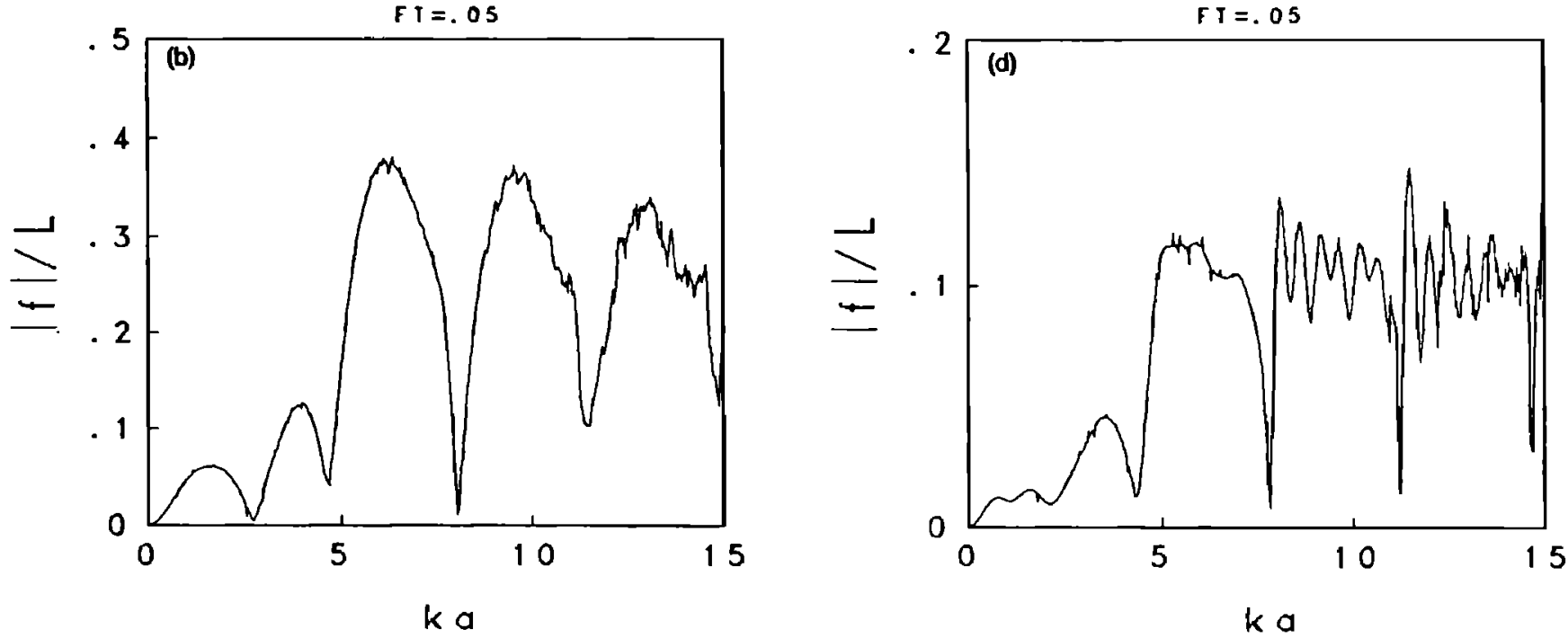

FIG. 8. Expanded plots from bottom row of Fig. 4 on linear scales.

ducers so that the tube was bent symmetrically away from the transducers.

Final alignment of the objects was done acoustically so that normal incidence backscatter measurements could be performed. The alignment procedure has to be done in an iterative manner because, if one naively aligns the objects so that the echo is at a maximum at one frequency, the object is not necessarily normal to the direction of the incident beam. This is due to the irregular shape of the backscattering cross section versus $k a$ curve in the $k a>1$ region (see figures in Sec. II). If one coincidentally is at a frequency such that the normal incidence cross section is at a local maximum, then a maximum echo will correspond to normal incidence alignment. However, if one is away from a local maximum and in fact in or near a sharp null, deviations in alignment away from normal incidence will modify the wave number according to the equation $K=k \sin \theta$ so that the backscattering cross section can actually increase away from normal incidence and produce a false indication of apparent normal incidence. Thus we first performed the backscatter measurements over the entire range of frequencies with the object "aligned" at an arbitrary frequency to determine the approximate location of the maxima of the curve and then repeated the measurements by aligning at a frequency that corresponded to a local maximum.

Because we used two transducers in the backscatter measurement, absolute calibration of the system was straightforward. After the backscatter measurements were 
performed, the transducers were separated by approximately $75 \mathrm{~cm}$ and aligned so that they would be aimed at each other. Because of the high gain of the power amplifier and preamplifier, the signal level was reduced by reducing the output of the signal generator (Hewlett-Packard 8116A) to prevent saturation of the received signal in the preamp. In this bistatic configuration, the system is linear below the saturation level so the reduction in signal level can be conveniently compensated for in the calibration equation. Using Eqs. (3) and (4), the equation used in the calibration is

$$
\sigma_{\text {bs }}=\left(V_{\text {scat }} / G V_{\text {cal }}\right)^{2}\left(r_{\text {scat }}^{4} / r_{\text {cal }}^{2}\right) \text {, }
$$

where $V_{\text {scat }}$ is the voltage amplitude of the backscattered signal, $V_{\text {cal }}$ is the voltage amplitude of the calibration signal, $G$ is the factor to compensate for the reduction in signal level for the calibration (for example, if the signal level was reduced by a factor of 10 during calibration, then $G=10$ ), $r_{\text {scat }}$ is the distance between the transducer pair and tube during the backscatter measurements, and $r_{\text {cal }}$ is the distance between the two transducers during the calibration. The calibration was performed at each frequency that the scatter measurements were performed $(1-2.5 \mathrm{MHz}$ in $10-\mathrm{kHz}$ steps). The measured cross sections were finally normalized by the square of the length (or arc length) of the tubes for plotting.

\section{B. Data}

Figure 9 compares the backscatter data collected from the straight and uniformly bent stainless steel tubes and predicted levels based on the corresponding equations in Sec. I and material properties published by Neubauer. ${ }^{30}$ The data were calibrated using Eq. (16). Plotted is the backscattering cross section normalized by the square of the length (or arc length) of the tubes versus $k a$. The straight cylinder data were from the entire 1- to $2.5-\mathrm{MHz}$ frequency range. However, because the backscatter levels from the bent cylinder are inherently lower, the echoes in much of the frequency range were below the noise level of the system (the system was more sensitive at the upper end of the frequency range where the resonance of the transducers occurs). As a result, only echoes from the upper portion of the frequency range were observable for the bent cylinder. There are certainly methods to enhance the signal-to-noise ratio of the lower frequency data, such as use of a digital oscilloscope for signal

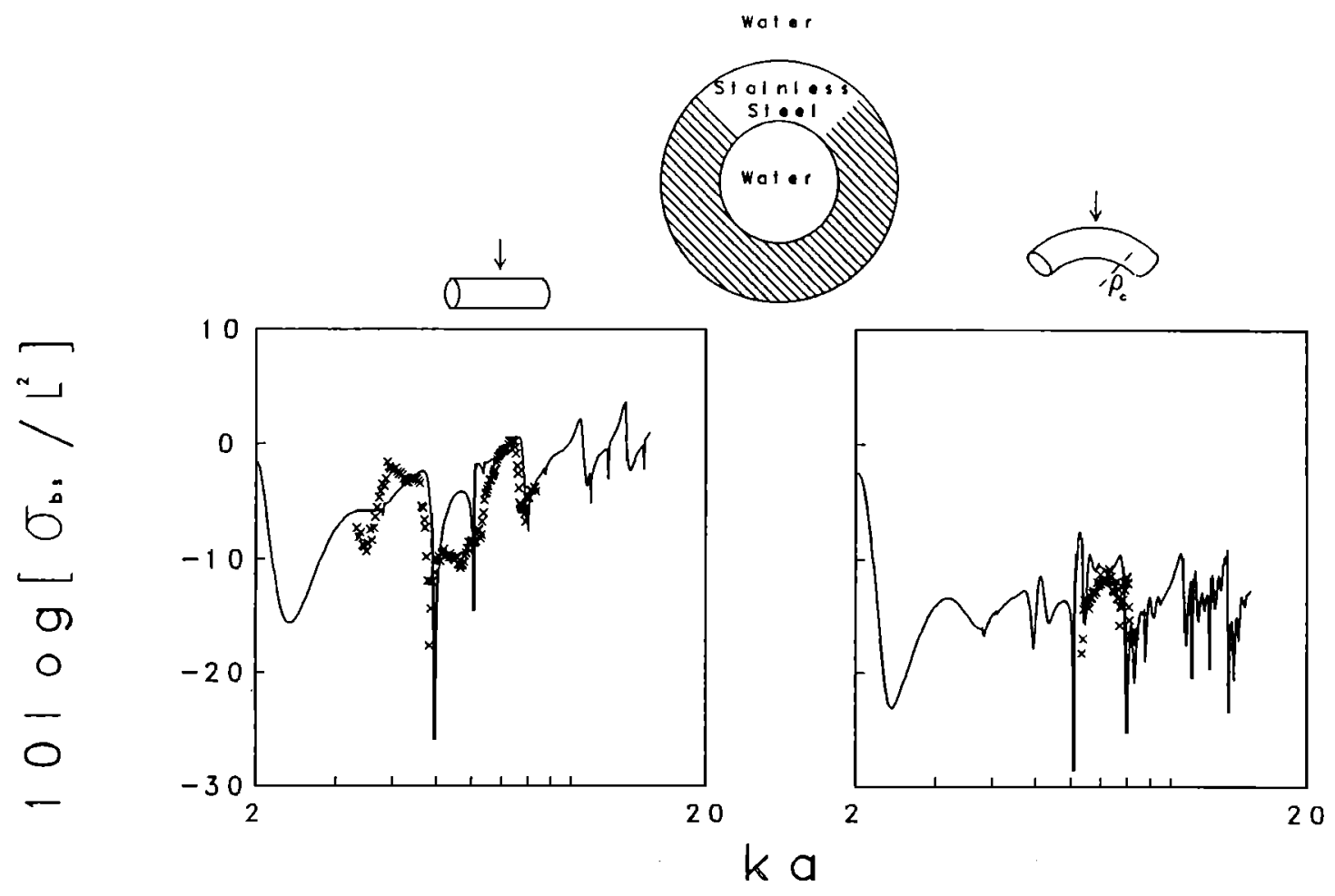

- theory

$x$ experi ment

FIG. 9. Comparison between deformed cylinder backscattering predictions and laboratory data involving stainless steel tubes immersed in tap water. The dramatic changes in structure and overall levels $(\sim 12 \mathrm{~dB})$ due to the bending of the tube are illustrated. Both tubes were 4.8 mm long by $1 / 16$-in. outside diameter (with an 0.03 -in. inside diameter the fractional thickness of each tube was 0.52 ). The ratio of length to outside diameter of each tube was 3.02 while the ratio of radius of curvature to arc length of the bent tube was 1.25 . 
averaging or use of a computer-based data acquisition system and signal filtering and averaging in the software, but that was beyond the scope of these early measurements.

There is reasonable correlation between the structure of the predicted and measured cross sections. The position of most of the observed major peaks and nulls are predicted by the theory with the exception of the data occurring at the low end of each respective frequency range. As discussed above, the sensitivity of the system increased with increasing $k a$ up to the resonance frequency of the transducers and hence the data quality is relatively poor at the lowest frequencies. There is very good correlation between the observed and predicted position of the nulls at $k a \sim 5$ and 8 for the straight cylinder. The lowest data point on the $k a \sim 5$ null corresponds to the noise level at that frequency; hence, the actual point is lower. The observed "shoulder" at values of $k a$ just above the $k a \sim 5$ null is much lower than the predicted level. A replicate of this measurement, not given, also indicates the presence of these lower levels, thus there is a clear discrepancy between predictions and observed values at $k a \sim 6$.

There are many possibilities for error both in the measurements and in the predictions. Predictions: (a) lack of knowledge of exact material properties. The predictions rely intimately on knowledge of the material properties such as density and compressional and shear speeds of sound. Because of the small size of the objects, it was impossible to even attempt measurement of the properties and hence, it was necessary to take values published in the literature. There were differences of the order $6 \%$ between the values of speed of sound given by Kaye and Laby ${ }^{35}$ and Neubauer. ${ }^{30}$ This may be due to differences in annealing processes. In attempting to use the Kaye and Laby parameters, there were dramatic differences between those curves and the ones given in this article - especially with respect to the positions of the peaks and nulls. Thus this author believes one major source of error in this comparison is the potential use of incorrect material properties. (b) The model ignores end effects. Since these objects are of moderate aspect (ratio of length to diameter is approximately 3 ). There should not be substantial errors introduced by the approximation at normal incidence. A recent study shows errors in the deformed cylinder approach to be 0.9 and $0.3 \mathrm{~dB}$ for aspect ratios of 2 and 5 , respectively, when compared with the exact solution to the rigid and fixed prolate spheroid..$^{24}$ Thus it is possible that the approximation could cause minor deviations, both in the overall levels and possibly in the finer highlights of the structure of the plot. Measurements: (a) as in any experiment, there is a degree of error associated with the measurements, ranging from finite signal-to-noise ratio of the electrical signals to errors in measuring the length of each tube. We had special difficulty in constructing a tube that was bent uniformly and furthermore it was difficult in measuring its radius of curvature. (b) As mentioned above, we had difficulty in aligning the objects in the acoustic beam, especially the straight tubes (since the scattering by bent objects is dominated by the first Fresnel zone of the bent objects, ${ }^{24}$ alignment of the bent tube was not a major problem). It is quite possible that the alignment was done slightly off a major maximum in the backscatter curve (see above discus- sion) and the measurements were performed slightly off 90 deg. Such an error would cause a shifting in the data toward higher $k a$ values.

In conclusion, the degree of agreement between the predictions and data suggests the validity of the models, at least within the range of conditions of the experiment. Also the data, as do the predictions, demonstrate the dramatic differences ( $\sim 12 \mathrm{~dB}$ in this case) between the scattering by straight and curved objects.

\section{CONCLUSIONS}

With the newly developed deformed cylinder solution, we were able to estimate the scattered fields due to elastic shelled bodies with the shapes of the prolate spheroid, straight finite cylinder, and uniformly bent finite cylinder. The elongated objects all had high aspect ratios. The scattered fields due to the elongated objects were compared with the exact solution to the spherical shell in a number of numerical simulations involving fluid-filled and gas-filled elastic shelled bodies with a wide range of shell thicknesses (all surrounded by fluid). The simulations illustrate the strong dependence of the scattering structure (overall level, trends, and positions of nulls and peaks) upon body size, shape, composition, and shell thickness. Comparisons were also made between straight and bent cylinder calculations and laboratory data (3:1 aspect ratios and 52\% shell thicknesses). There was reasonable agreement between the predictions and data although the comparison was limited by lack of knowledge of the exact material properties for use in the predictions. In addition, the data illustrated the dramatic change in backscattering cross section when the cylinder was bent $(\sim 12 \mathrm{~dB})$.

The comparisons between the deformed cylinder theory and data show promise in use of the approximate theory under certain conditions such as with high aspect objects at or near broadside incidence. Because of its increase in accuracy as the aspect ratio increases and relatively low computational times, the theory is an attractive complement to other methods that are practical only for lower aspect ratios.

The purpose of this research was to produce a theoretical and numerical foundation for the description of the scattering of sound by elongated marine organisms that have shells. Much work remains to be done in that area such as measuring the material properties of the shell of the organisms and inner body. Furthermore, the organisms are not perfectly described by simple shapes such as uniformly bent cylinders or prolate spheroids. However, now there is a foundation for proceeding to the next step of describing the scattering by more complex shapes.

\section{ACKNOWLEDGMENTS}

The author is grateful to the following people from the Woods Hole Oceanographic Institution, Woods Hole, MA: Bob Eastwood for both his aid in the numerical computations on the computer and assistance in setting up the scattering experiment and Shirley Bowman for preparing the manuscript for this article. The author is also indebted to the following WHOI 1989 summer student fellows: Doron 
Weisbarth (Trenton State College, Trenton, NJ) for performing the measurements, and Shabnam Merchant (Bryn Mawr College, Bryn Mawr, PA) for donating samples of her hair throughout the summer of 1989 for our mounts for the tubes. This work was supported in part by the U.S. Office of Naval Research. This is Woods Hole Oceanographic Institution Contribution No. 7228.

' R. R. Goodman and R. Stern, "Reflection and transmission of sound by elastic spherical shells," J. Acoust. Soc. Am. 34, 338-344 (1962).

${ }^{2} \mathrm{~S}$. Machlup, "A theoretical model for sound scattering by marine crustaceans," J. Acoust. Soc. Am. 24, 290-293 (1952).

${ }^{3} \mathrm{R}$. Hickling, "Analysis for echoes from a hollow metallic sphere in water," J. Acoust. Soc. Am. 36, 1124-1137 (1964).

4J. D. Murphy, J. George, A. Nagl, and H. Überall, "Isolation of the resonant component in acoustic scattering from fluid-loaded elastic spherical shells," J. Acoust. Soc. Am. 65, 368-373 (1979).

"A. J. Poggio, "Acoustic scattering," M. B. Associates Tech. Rep., San Ramon, CA. 94583 (1969).

'M. C. Junger, "Sound scattering by thin elastic shells," J. Acoust. Soc. Am. 24, 366-373 (1952).

'R. D. Doolittle and H. Überall, "Sound scattering by elastic cylindrical shells," J. Acoust. Soc. Am. 39, 272-275 (1966).

${ }^{8} \mathrm{P}$. Uginčius and $\mathrm{H}$. Überall, " Creeping-wave analysis of acoustic scattering by elastic cylindrical shells," J. Acoust. Soc. Am. 43, 1025-1035 (1968).

${ }^{9}$ K. J. Diercks,T. G. Goldsberry, and C. W. Horton, "Circumferential waves in thin-walled air-filled cylinders in water," J. Acoust. Soc. Am. 35, 59-64 (1963).

${ }^{10} \mathrm{G}$. C. Gaunaurd, "Sonar cross section of a coated hollow cylinder in water," J. Acoust. Soc. Am. 61, 360-368 (1977).

"C. W. Horton, W. R. King, and K. J. Diercks, "Theoretical analysis of the scattering of short acoustic pulses by a thin-walled metallic cylinder in water," J. Acoust. Soc. Am. 34, 1929-1932 (1962).

${ }^{12}$ G. C. Gaunaurd, "High-frequency acoustic scattering from submerged cylindrical shells coated with viscoelastic absorbing layers," J. Acoust. Soc. Am. 62, 503-512 (1977).

${ }^{13}$ L. Flax and W. G. Neubauer, "Acoustic reflection from layered elastic absorptive cylinders," J. Acoust. Soc. Am. 61, 307-312 (1977).

${ }^{14}$ S. Baskar, V. V. Varadan, and V. K. Varadan, "Thin shell theories and acoustic wave scattering by infinitely long cylindrical shells of arbitrary cross section," J. Acoust. Soc. Am. 75, 1673-1679 (1984).
${ }^{15}$ D. W. Brill, P. Uginčius, J. George, F. S. Chwieroth, and H. Überall, "Sound scattering from thin shells in the Kirchhoff approximation," J. Acoust. Soc. Am. 62, 1367-1372 (1977).

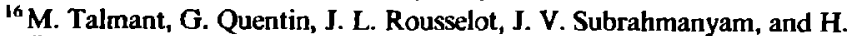
Überall, "Acoustic resonances of thin cylindrical shells and the resonance scattering theory," J. Acoust. Soc. Am. 84, 681-688 (1988).

${ }^{17}$ G. C. Gaunaurd and M. F. Werby, "Resonance response of submerged, acoustically excited thick and thin shells," J. Acoust. Soc. Am. 77, 20812093 (1985).

${ }^{18}$ B. Peterson, V. V. Varadan, and V. K. Varadan, "Scattering of acoustic waves by layered elastic and viscoelastic obstacles in water," J. Acoust. Soc. Am. 68, 673-685 (1980).

${ }^{19}$ R. H. Hackman and D. G. Todoroff, "An application of the spheroidalcoordinate-based transition matrix: The acoustic scattering from high aspect ratio solids," J. Acoust. Soc.Am. 78, 1058-1071 (1985).

${ }^{20} \mathrm{M}$. F. Werby and G. Gaunaurd, "Classification of resonances in the scattering from submerged spheroidal shells insonified at arbitrary angles of incidence," J. Acoust. Soc. Am. 82, 1369-1377 (1987).

${ }^{21}$ R. H. Hackman, personal communication (1989).

${ }^{22}$ T. K. Stanton; "Sound scattering by cylinders of finite length. I. Fluid cylinders," J. Acoust. Soc. Am. 83, 55-63 (1988).

${ }^{23}$ T. K. Stanton, "Sound scattering by cylinders of finite length. II. Elastic cylinders," J. Acoust. Soc. Am. 83, 64-67 (1988).

${ }^{24} \mathrm{~T}$. K. Stanton, "Sound scattering by cylinders of finite length. III. Deformed cylinders," J. Acoust. Soc. Am. 86, 691-705 (1989).

${ }^{25}$ T. K. Stanton, "Simple approximate formulas for backscattering of sound by spherical and elongated objects," J. Acoust. Soc. Am. 86, 1499-1510 (1989).

${ }^{26} \mathrm{C}$. S. Clay and H. Medwin, Acoustical Oceanography: Principles and Applications (Wiley-Interscience, New York, 1977).

${ }^{27}$ R. J. Urick, Principles of Undenwater Sound (McGraw-Hill, New York, 1983).

${ }^{28}$ E. Skudrzyk, The Foundations of Acoustics (Springer, New York, 1971). ${ }^{29}$ Handbook of Mathematical Functions, edited by M. Abramowitz and I. A. Stegun (Dover, New York, 1970).

${ }^{30}$ W. G. Neubauer (Author/Ed.) Acoustic Reflection from Surfaces and Shapes (Naval Research Laboratory, Washington, DC).

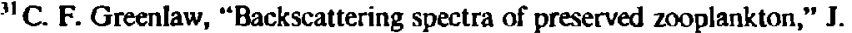
Acoust. Soc. Am. 62, 44-52 (1977).

${ }^{32}$ J. J. Faran, Jr., "Sound scattering by solid cylinders and spheres," J. Acoust. Soc. Am. 23, 405-418 (1951).

${ }^{33}$ V. C. Anderson, "Sound scattering from a fluid sphere," J. Acoust. Soc. Am. 22, 426-431 (1950).

${ }^{14} \mathrm{H}$. Überall, "Surface waves in acoustics," in Physical Acoustics, edited by W. P. Mason and R. N. Thurston (Academic, New York, 1973), Vol. 10, pp. $1-60$.

${ }^{35}$ G. W. C. Kaye and T. H. Laby, Tables of Physical and Chemical Constants (Longman, New York, 1973). 